\title{
Dynamical structures of interaction wave solutions for the two extended higher-order KdV equations
}

\author{
Zillur Rahman ${ }^{1}$, M. Zulfikar Ali ${ }^{2}$, Harun-Or- Roshid ${ }^{3}$, Mohammad Safi Ullah ${ }^{1}$, and \\ Xiao-Yong Wen ${ }^{4}$ \\ ${ }^{1}$ Comilla University \\ ${ }^{2}$ University of Rajshahi \\ ${ }^{3}$ Pabna University of Science and Technology \\ ${ }^{4}$ Beijing Information Science and Technology University,
}

June 27, 2020

\begin{abstract}
In this article, we study two extended higher-order KdV-type models, namely, the extended Sawada-Kotera (eSK) and the extended Lax (eLax) equations. These models successfully describe propagation of dimly nonlinear long waves in fluids, ionacoustic waves in harmonic sparklers. We firstly derive multi-soliton solutions of the models. We then construct interection solutions in-terms of hyperbolic and sinusoidal functions using the multi-soliton solutions with appropriate complex conjugate parameters. Such parameters influence and control the phase shifts, propagation direction and energies of the waves. In particularly, we present their collision solutions in the identical plane with different parametric constraints, which degenerate to the line rogue waves, $\mathrm{x}$-shaped rogue waves, cnoidal periodic waves, interactions of rogue and bell waves, line breather and double breather waves. The dynamical characteristics of the wave solutions has been plotted by choosing particular values of the parameters in graphically.
\end{abstract}

\section{Hosted file}

Article-19.06.2020.doc available at https://authorea.com/users/337342/articles/462942dynamical-structures-of-interaction-wave-solutions-for-the-two-extended-higher-orderkdv-equations 


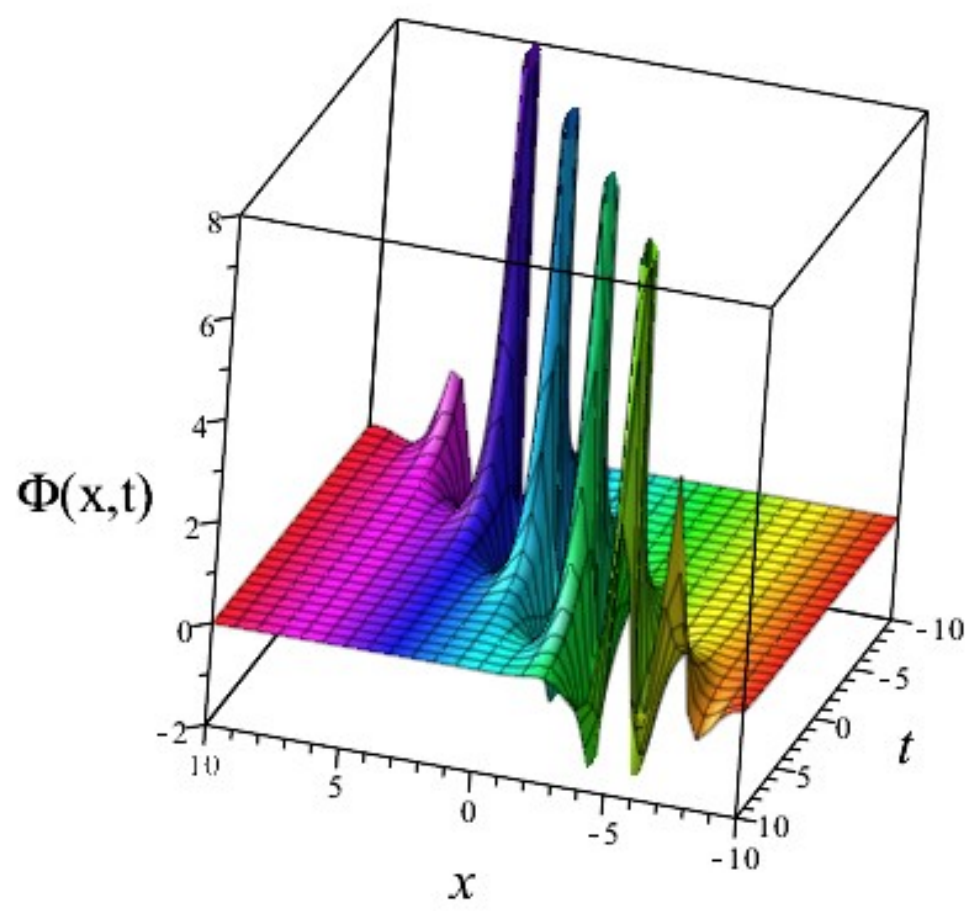





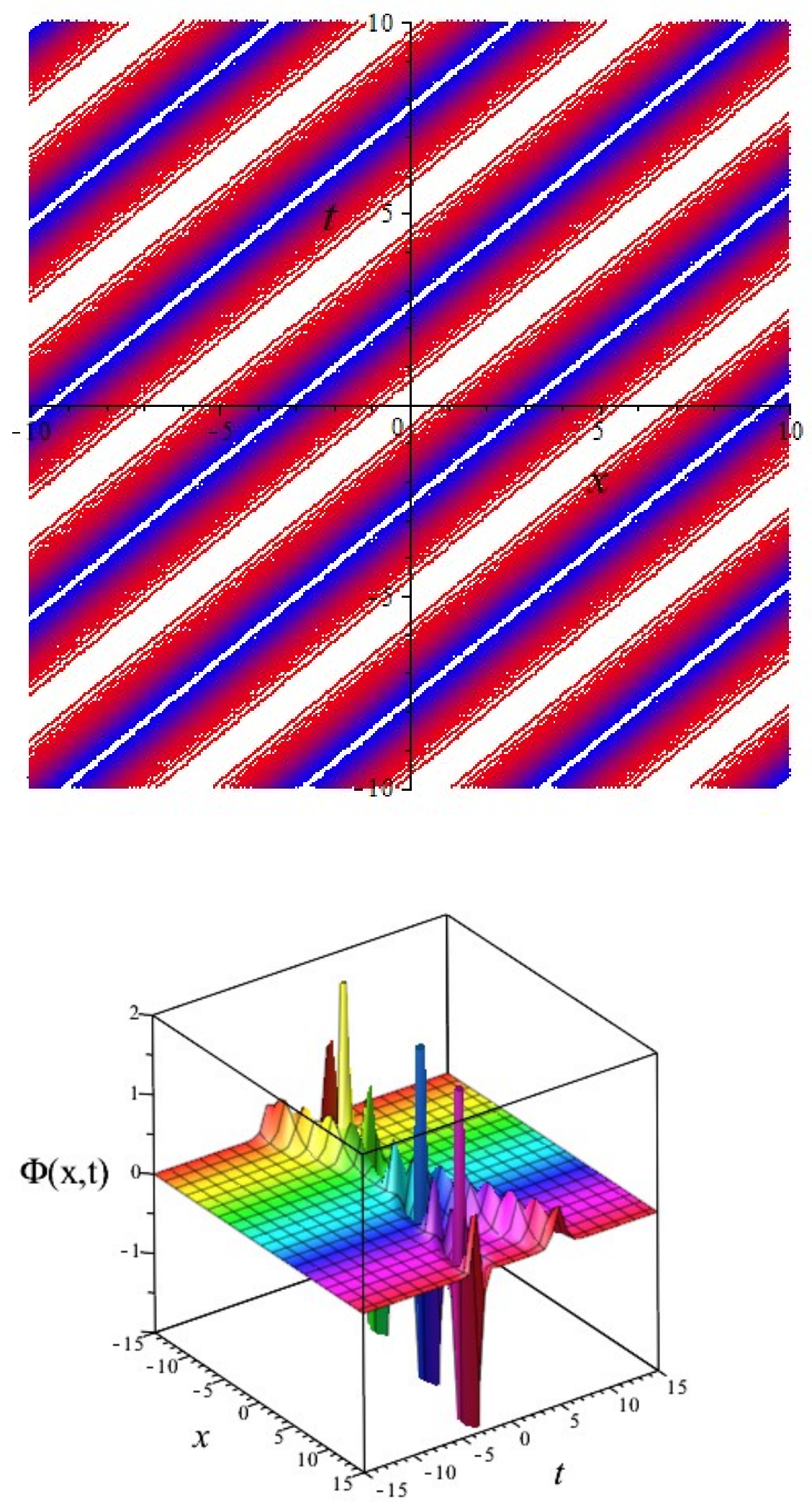


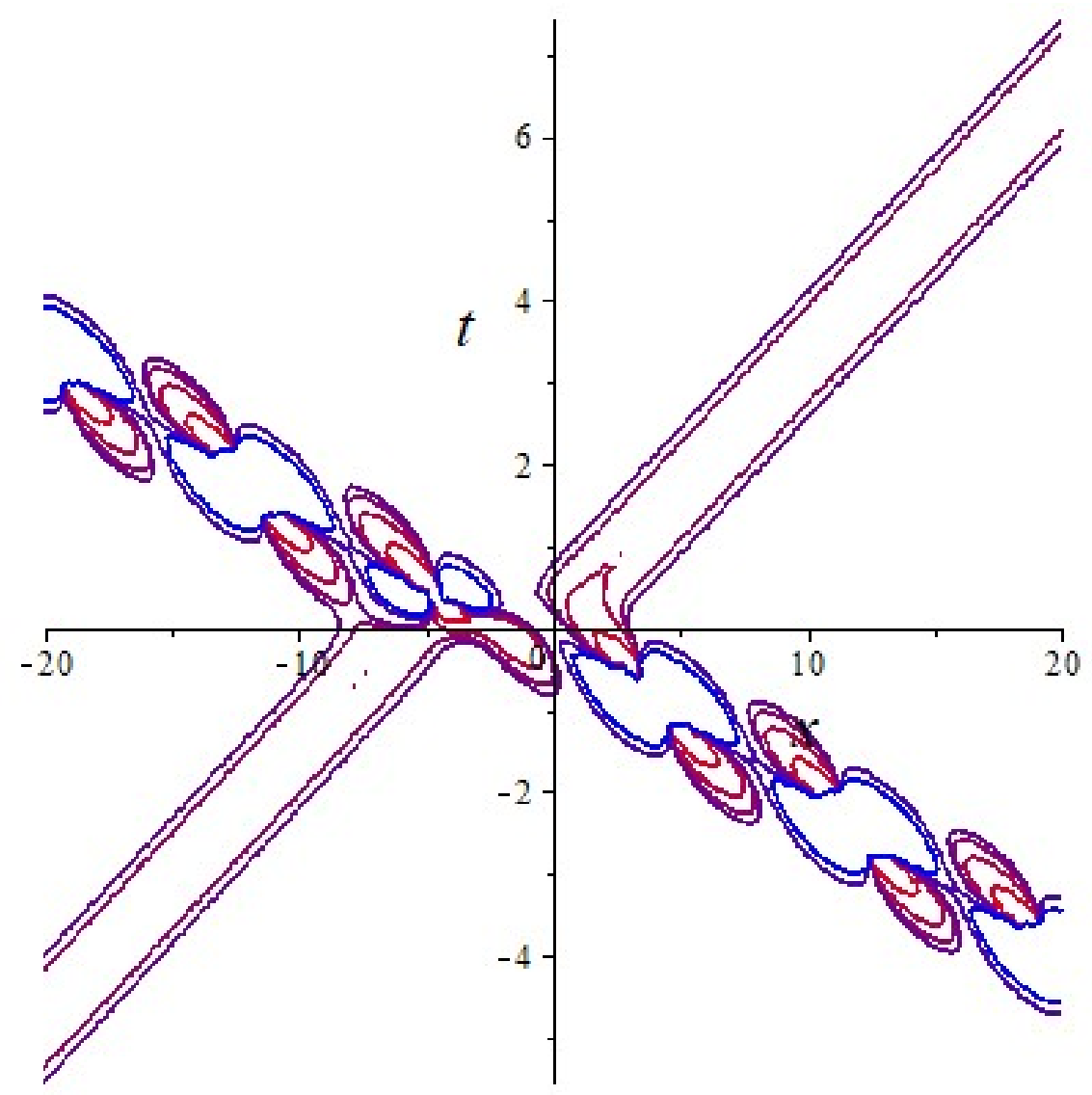




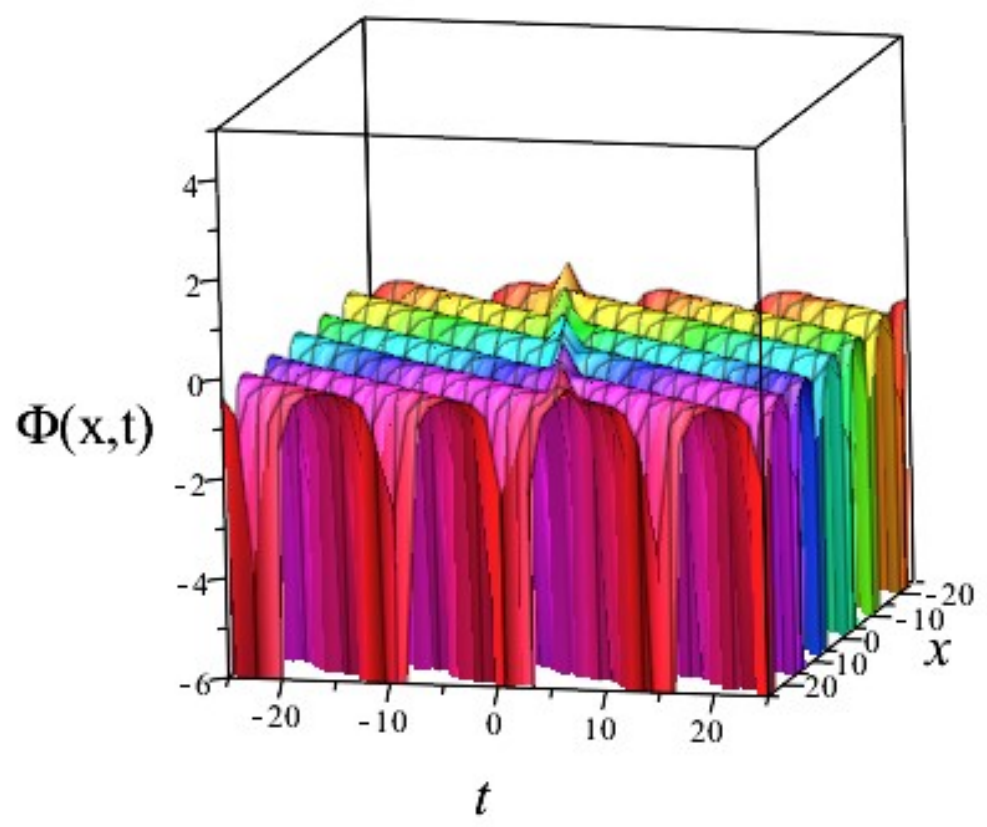



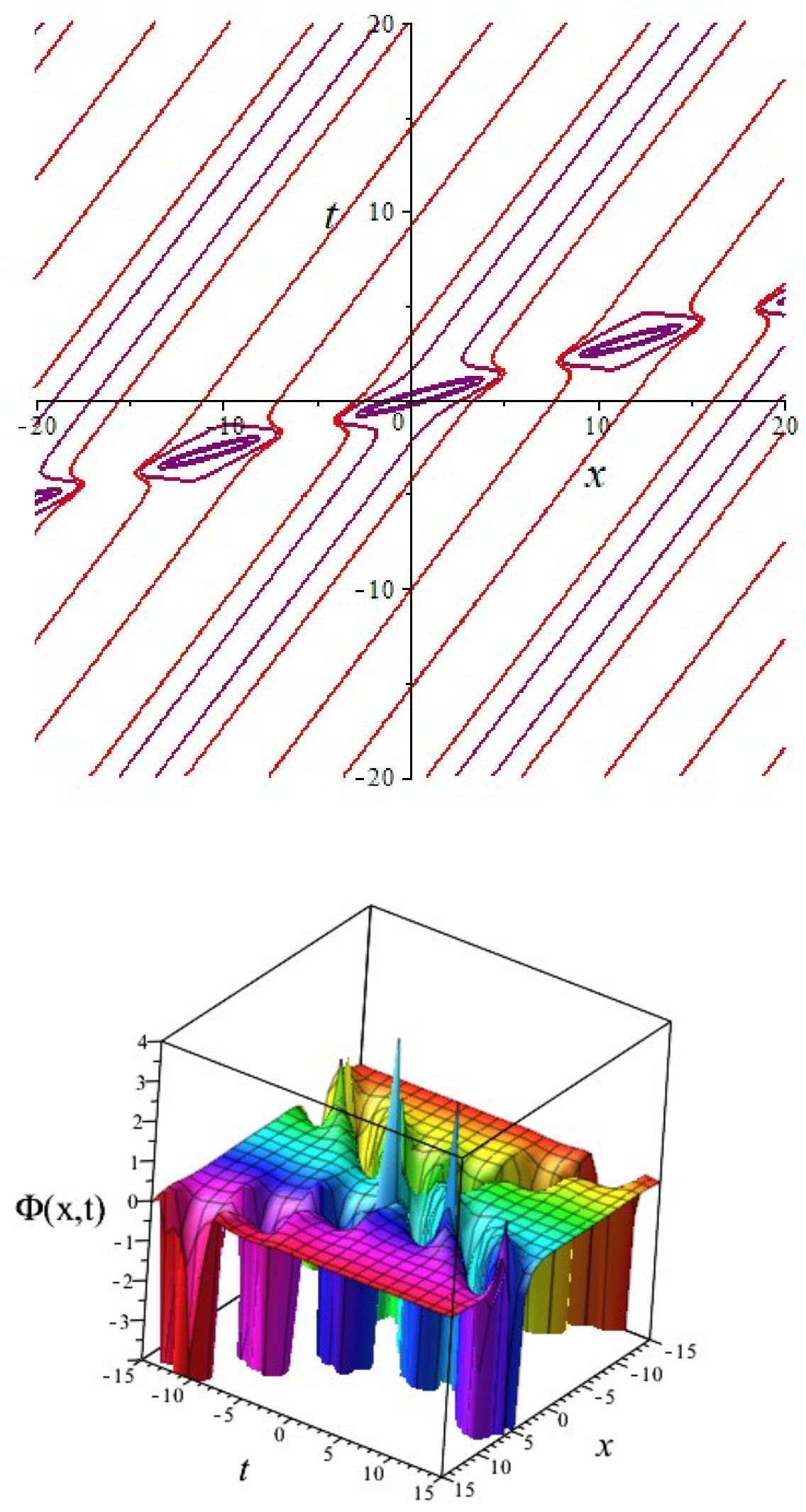

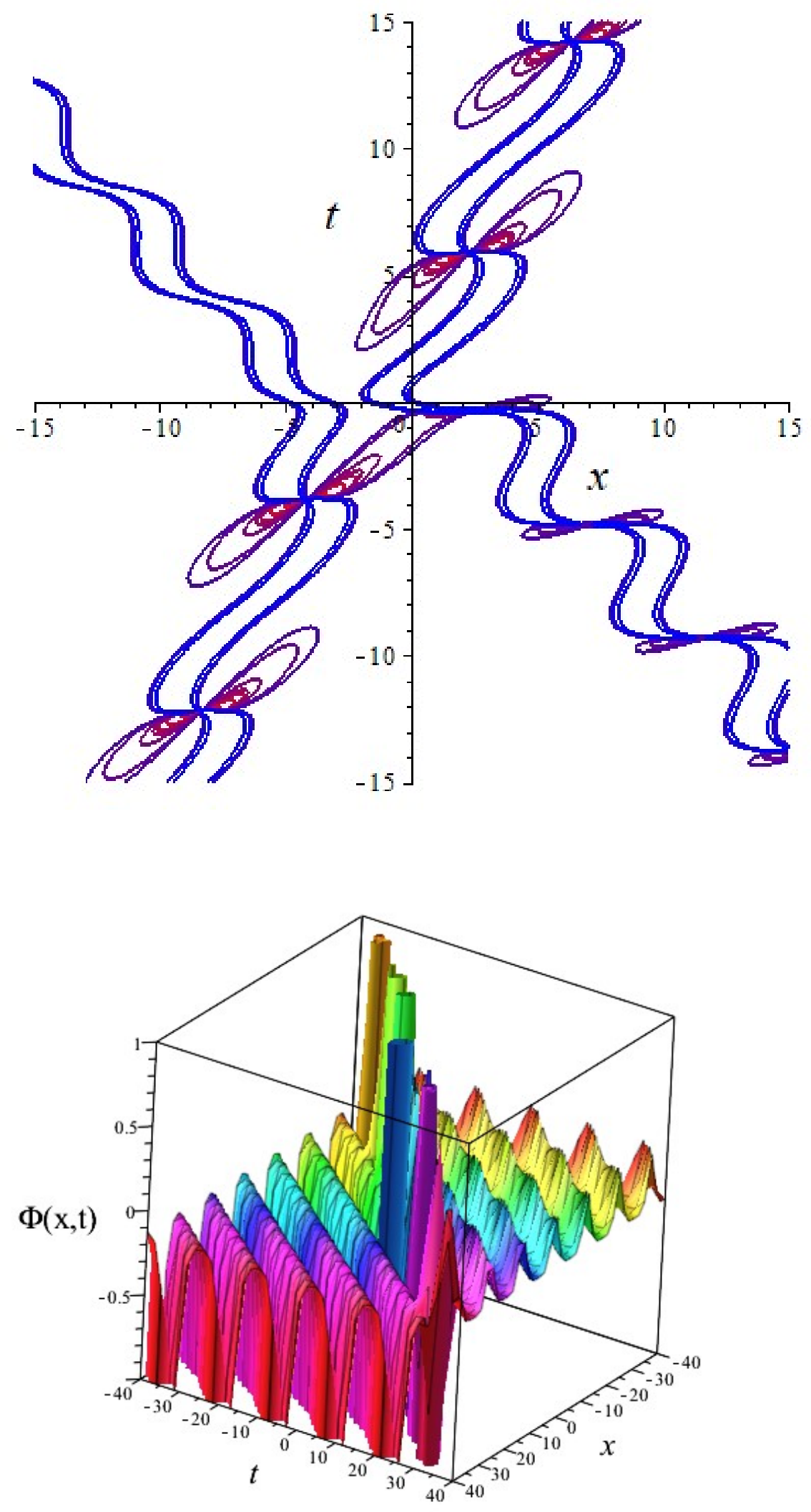









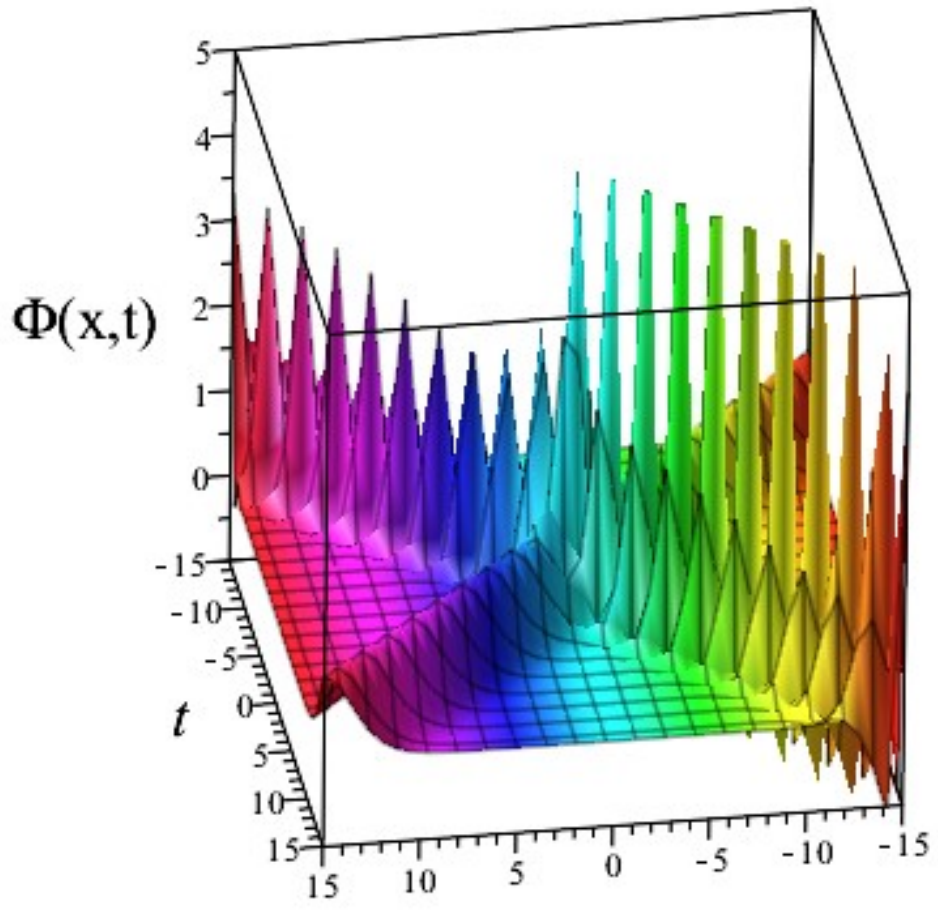



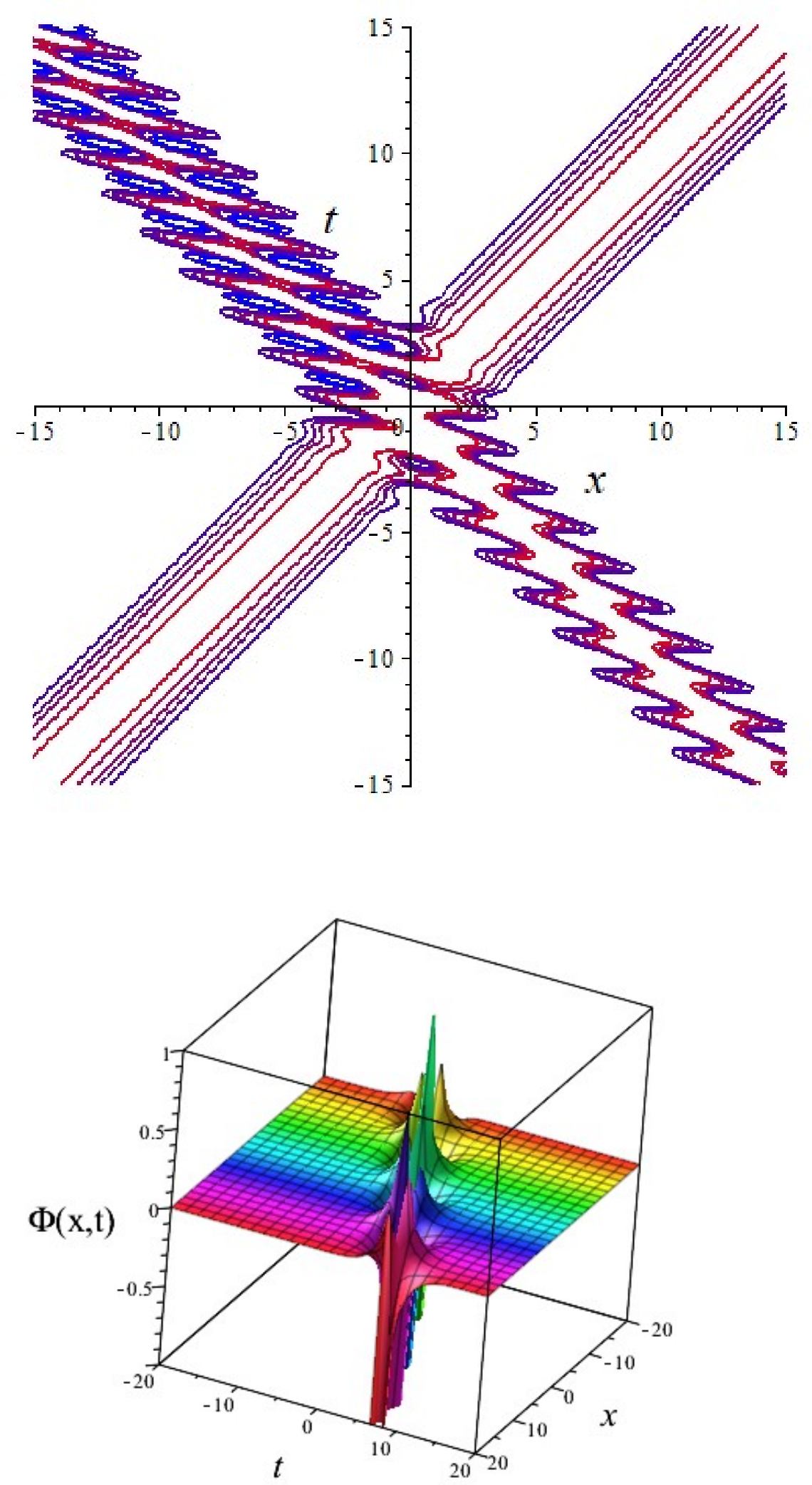


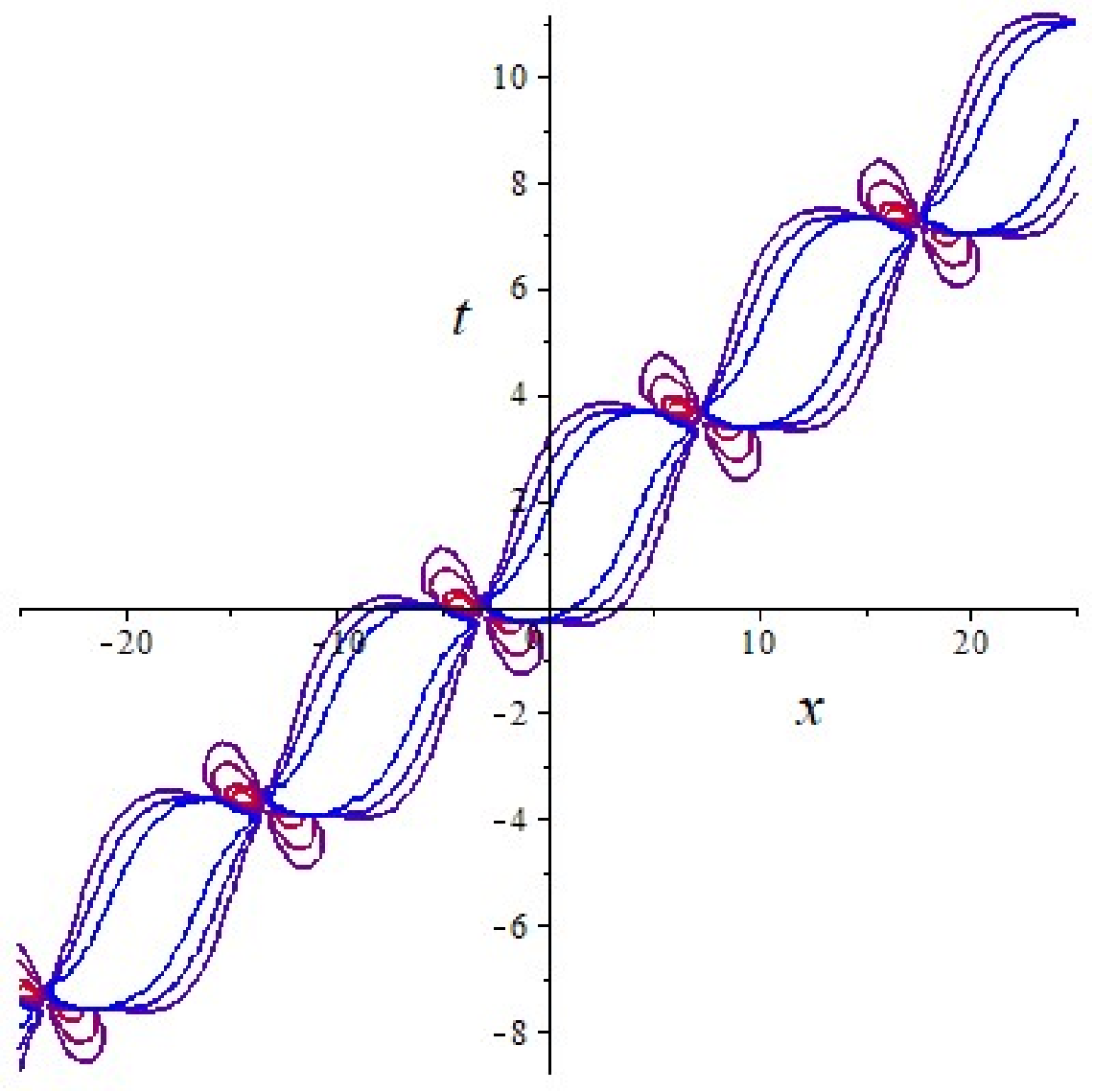




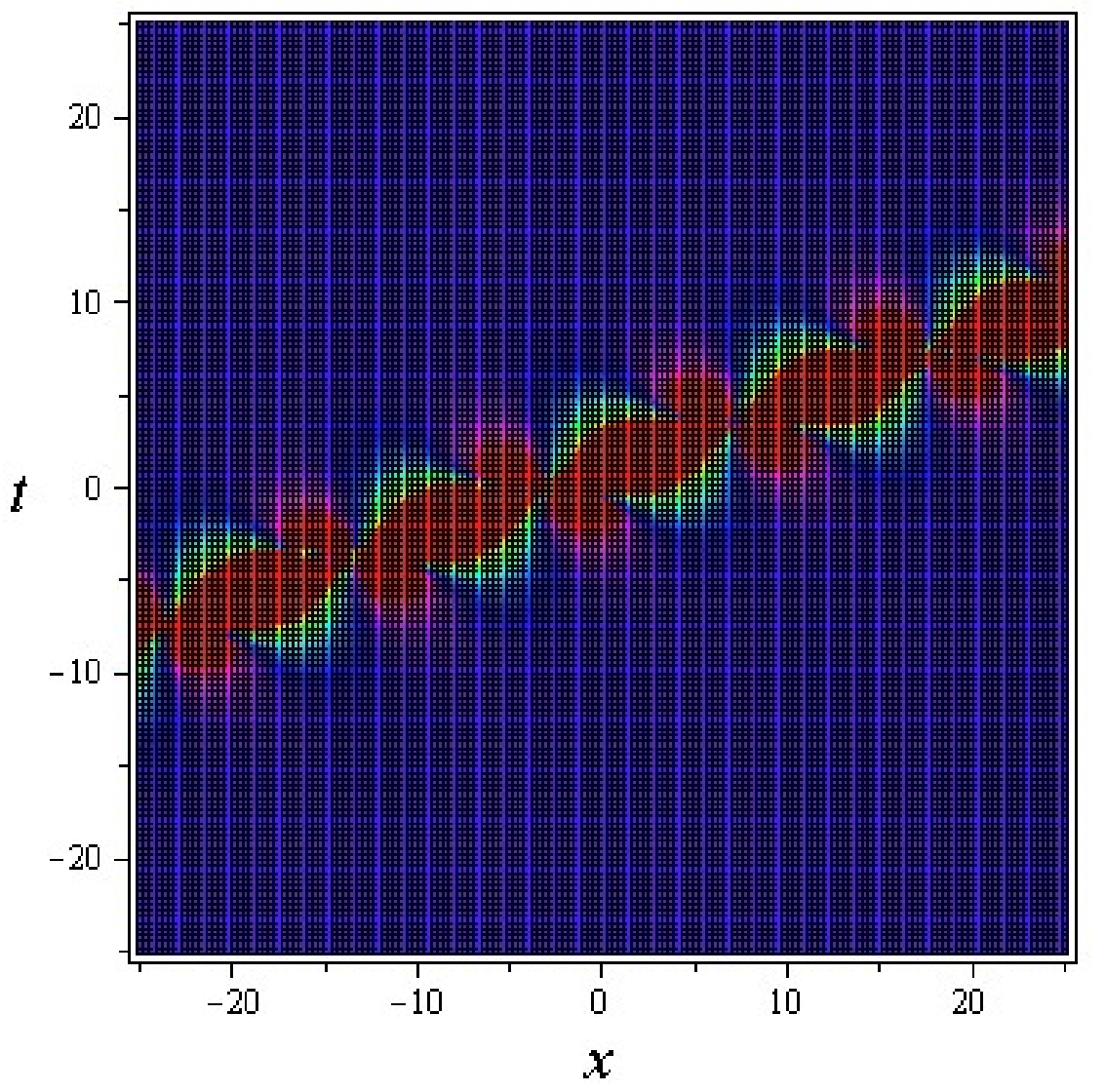



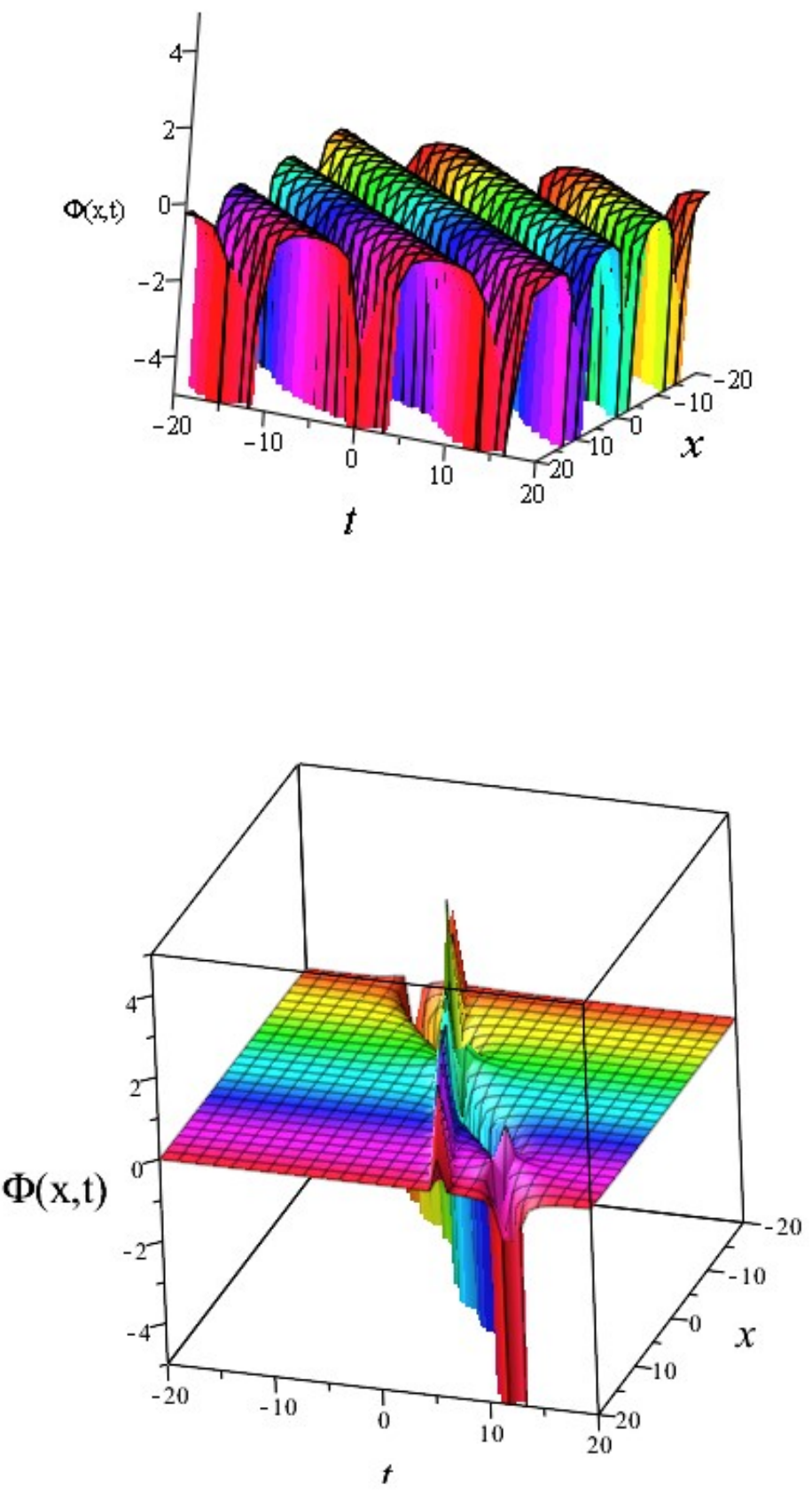

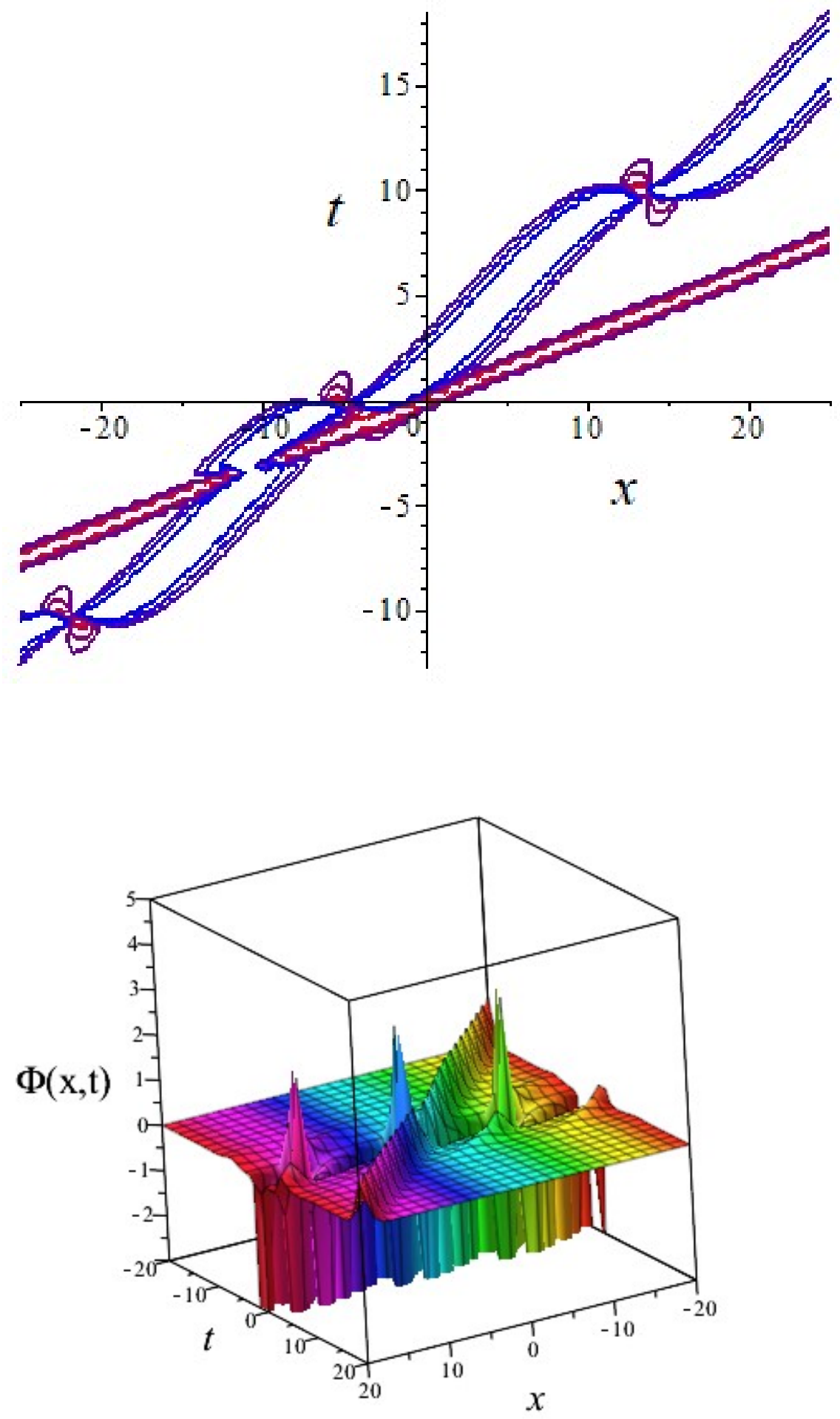

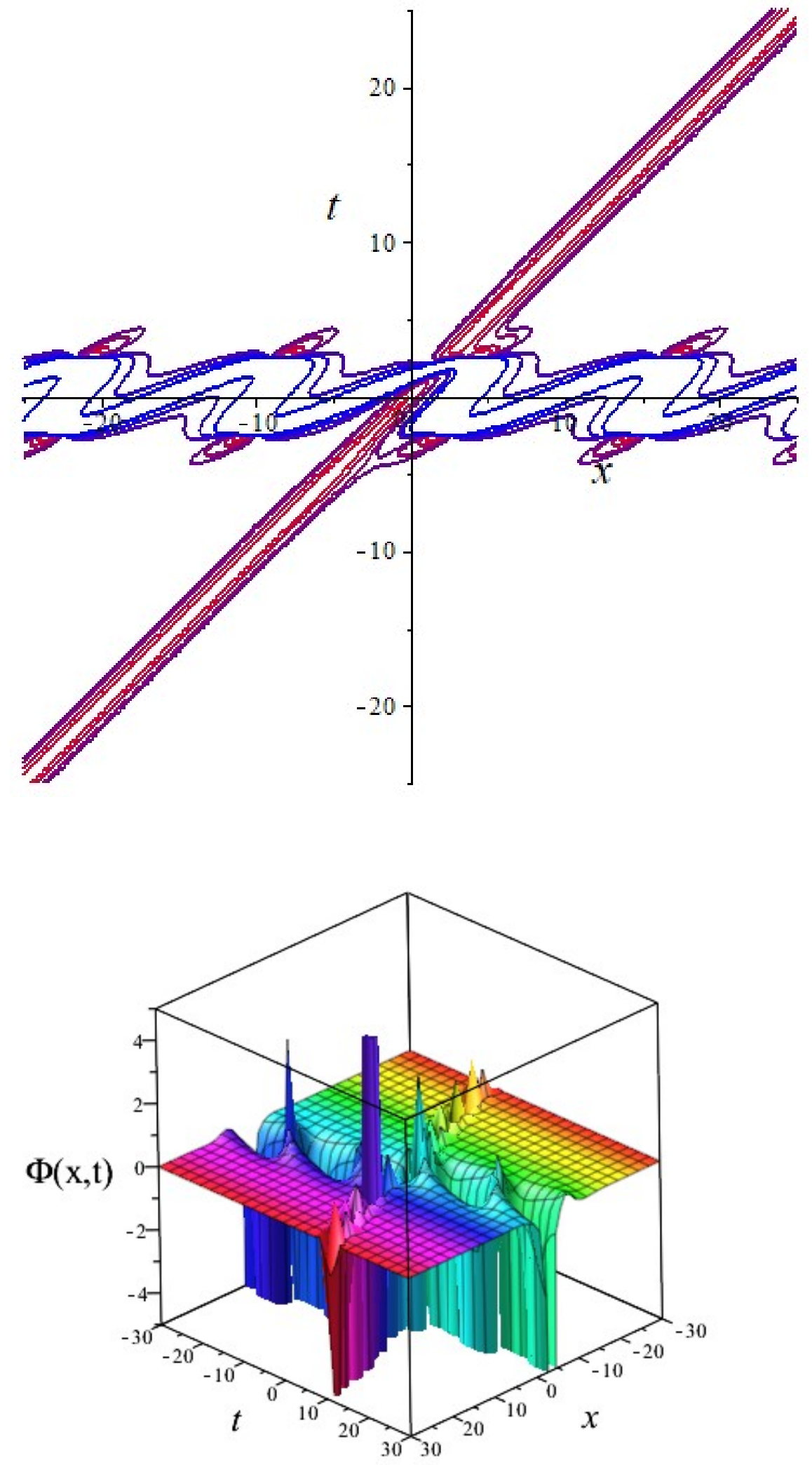


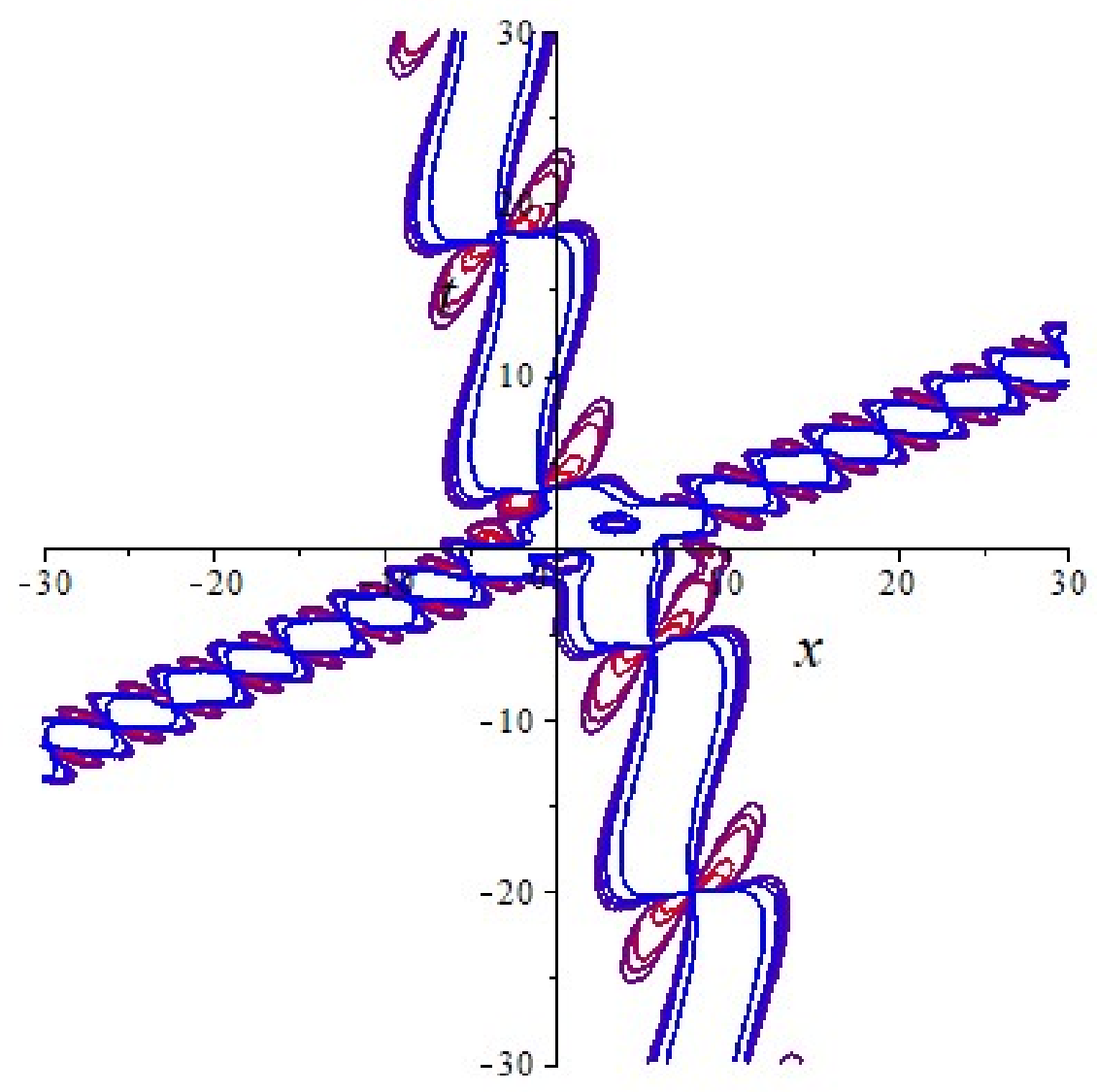








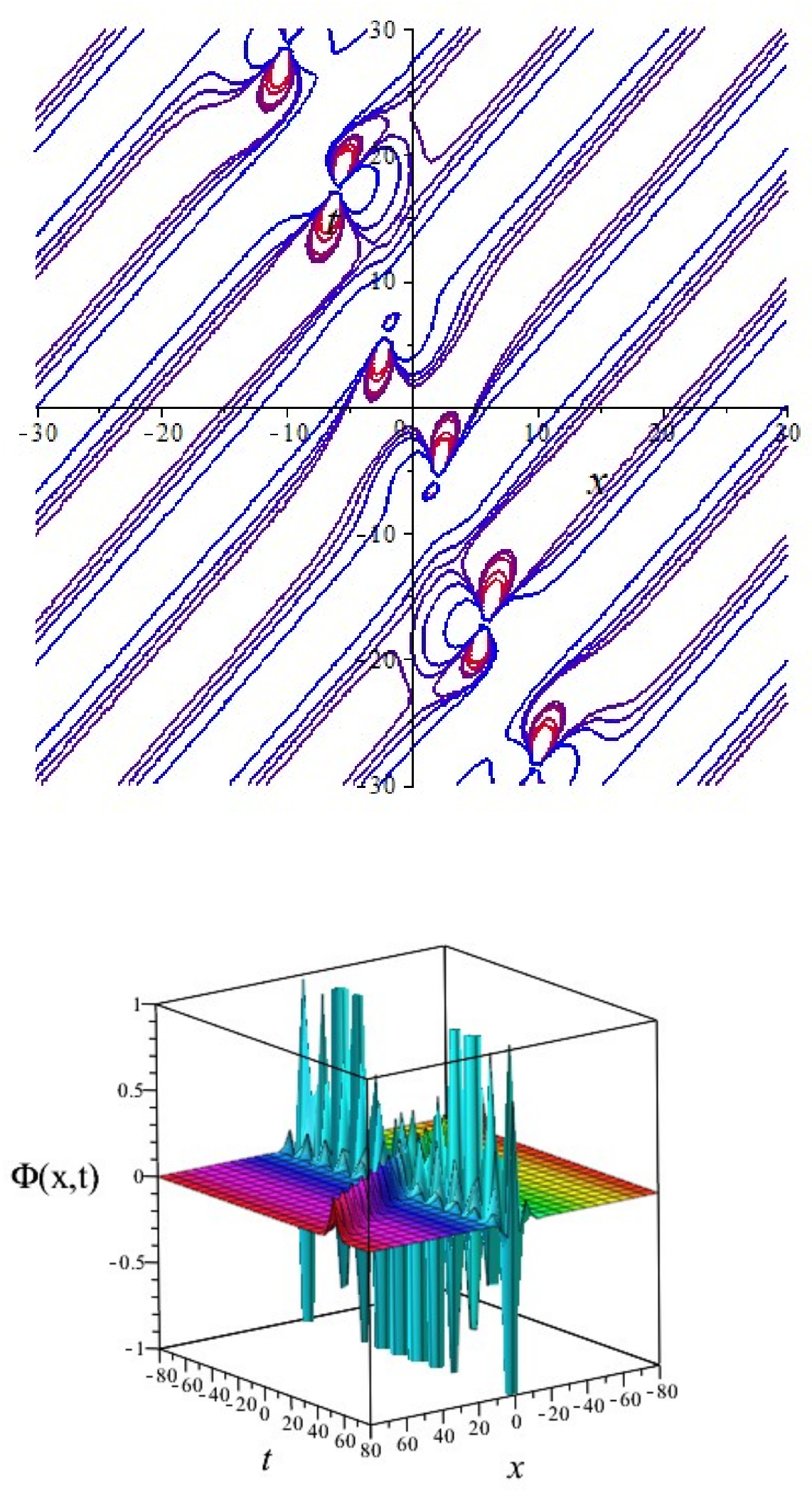

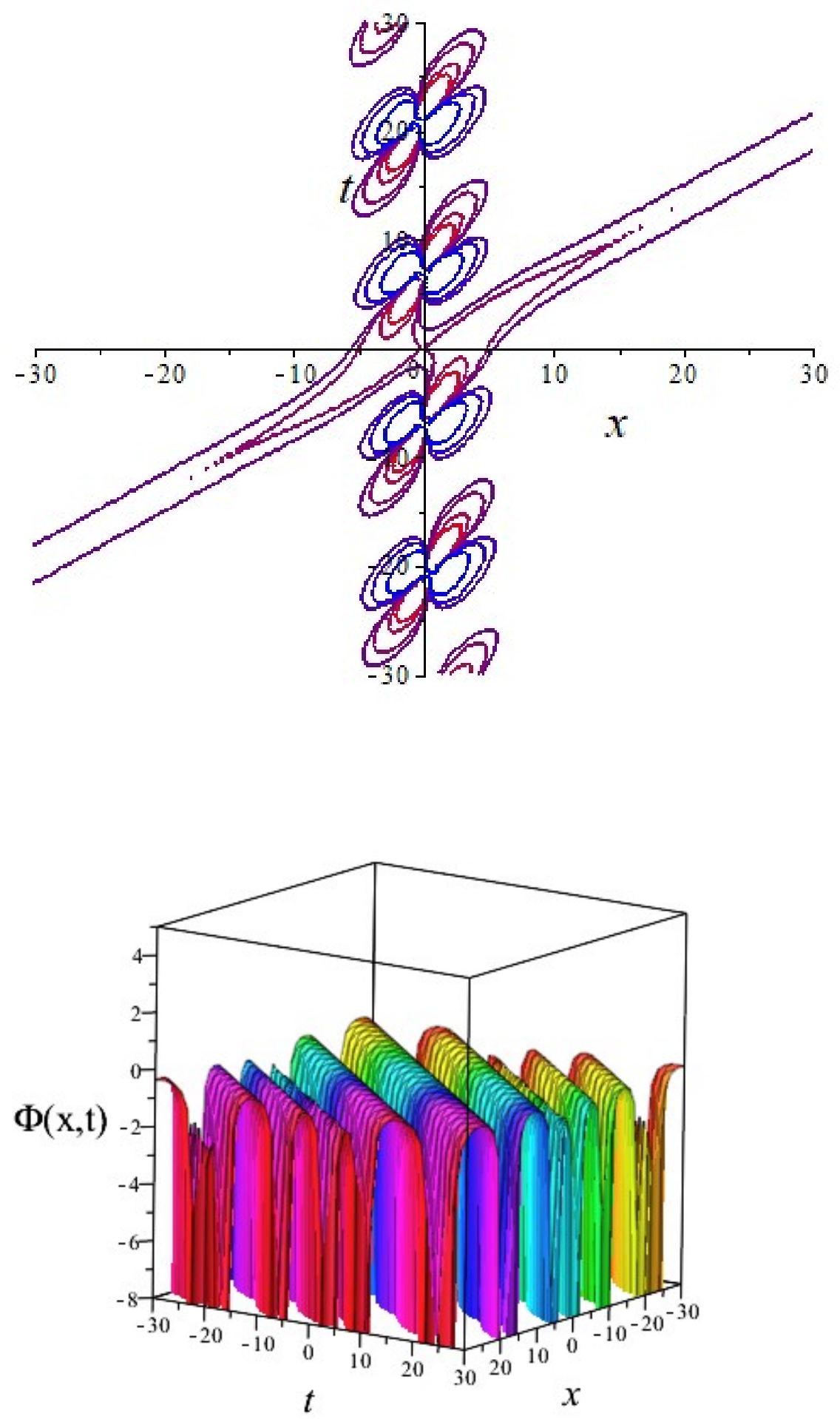


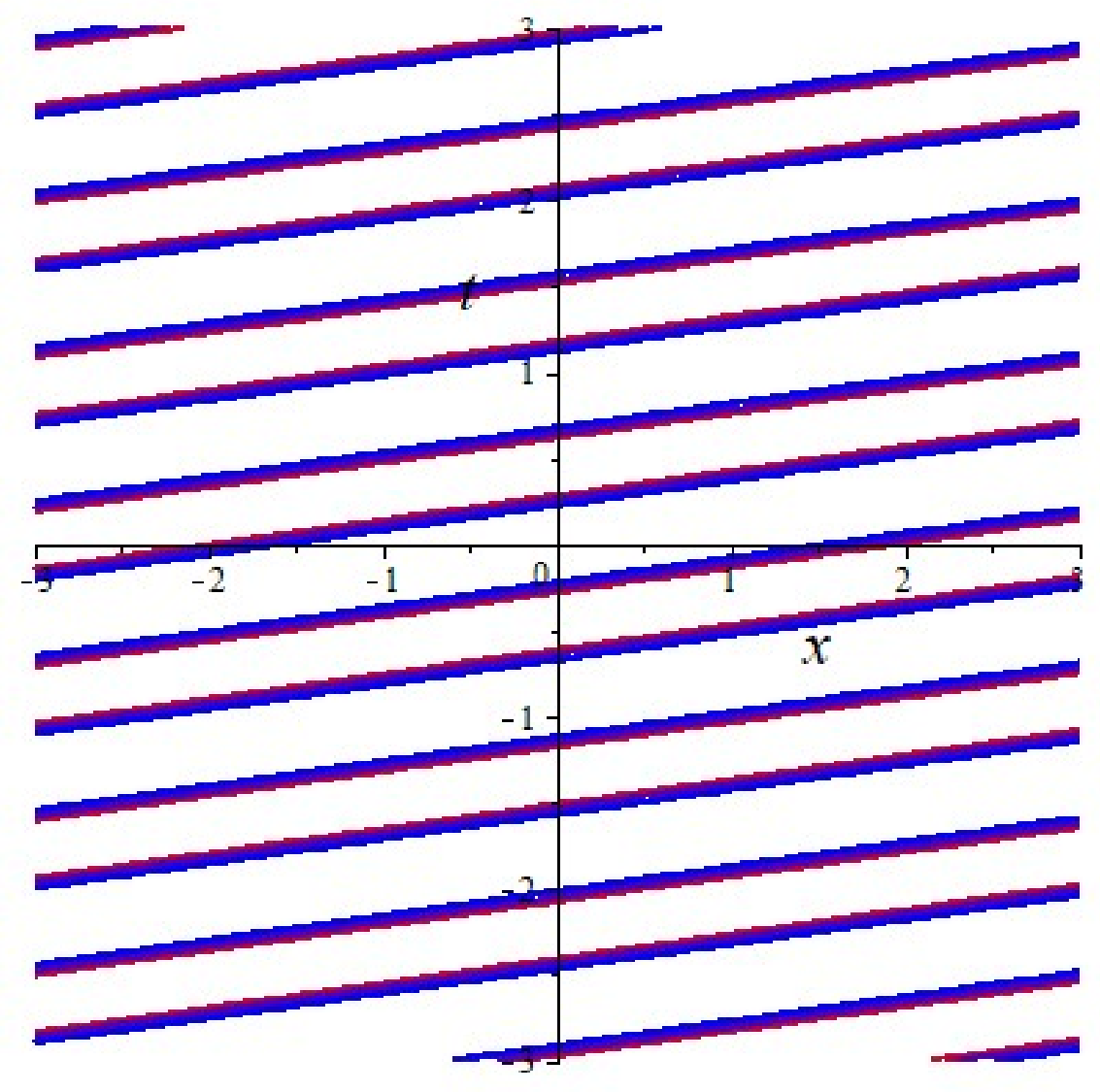

